\title{
Slow-light dispersion in coupled periodic waveguides
}

\section{Andrey A. Sukhorukov, ${ }^{1}$ Andrei V. Lavrinenko, ${ }^{1,2}$}

Dmitry N. Chigrin, ${ }^{3}$ Dmitry E. Pelinovsky, ${ }^{4}$ and Yuri S. Kivshar ${ }^{1}$

${ }^{1}$ Nonlinear Physics Centre and Centre for Ultra-high bandwidth Devices for Optical Systems (CUDOS), Research School of Physical Sciences and Engineering, Australian National University, Canberra, ACT 0200, Australia

${ }^{2}$ DTU Fotonik, Department of Photonics Engineering, NanoDTU, Technical University of Denmark, Building 345V, DK-2800 Kgs. Lyngby, Denmark ${ }^{3}$ Physikalisches Institut, Universität Bonn, Nussallee 12, D-53115 Bonn, Germany ${ }^{4}$ Department of Mathematics, McMaster University, Hamilton, Ontario, Canada L8S $4 K 1$

Periodic waveguides bring a new twist onto the typical waveguiding problems been intermediate case between photonic crystal waveguides and photonic wires or ridge waveguides. We develop an asymptotic theory applicable for a broad class of coupled periodic waveguide structures, and use the analytical expressions to identify the generic types of dispersion in the vicinity of a photonic band-edge, where the group velocity of light is reduced. We show that the dispersion can be controlled by the longitudinal shift between the waveguides. We also demonstrate through finite-difference time-domain simulations examples of spatial and temporal pulse dynamics in association with different types of slow-light dispersion.

(C) 2008 Optical Society of America

OCIS codes: (050.5298) Photonic crystals; (230.7370) Waveguides; (250.5300)

Photonic integrated circuits. 


\section{Introduction}

The physics and applications of slow light are attracting increasing attention in recent years. In particular, the strong reduction of the pulse velocity by orders of magnitude was demonstrated experimentally in periodically modulated photonic structures [1-7]. Flexible control over the light propagation in micro-scale structures may lead to the realization of ultracompact delay lines, with the additional capability of flexible pulse shaping. Indeed, the slow-light regime can be realized when the pulse spectrum is tuned close to the edge of a photonic band-gap, where the dispersion curves feature a turning point and the group velocity is reduced down to zero. On the other hand, in this spectral region the groupvelocity dispersion becomes significant, defining the reshaping of the propagating pulse [8]. It was suggested that the slow-light dispersion can be controlled in specially designed structures [9-11], with the possibilities of obtaining either quartic band-edges or split band-edges where slow-light states feature non-vanishing phase velocity. Most recently, it was demonstrated, based on the general symmetry analysis, that the latter regime corresponds to the appearance of two distinct slow-light modes, which group velocity is automatically matched in the same frequency region [12]. The simultaneous excitation and beating of such modes opens new possibilities for the spatio-temporal control of pulse propagation, including the operation of photonic-crystal couplers for slow-light pulses [12].

In this work, we present a general analysis for the realization of different slow-light dispersion regimes by selecting a longitudinal shift between directionally coupled periodic waveguides (as indicated by parameter $z_{0}$ in the examples of structures sketched in Fig. 1). The key possibilities of dispersion engineering with quadratic, quartic, or split band-edges are illustrated in Fig. 2. The important effect of the photonic structure symmetry on the mode dispersion and band-gap spectrum was recognized in previous studies of Bragg-grating couplers [13-17], photonic-crystal waveguides [12, 18], and coupled nanopillar waveguides [1922]. Our analytical expressions are valid for a broad range of photonic structures, uncovering the essential physics underlying the similarities of slow-light dispersion characteristics previously obtained in the framework of distinct analytical and numerical methods. We show that the most flexible control over the band-edge dispersion can be obtained when the longitudinal shift can be varied continuously. In two-dimensional photonic-crystals, the shift may be constrained to particular values by the symmetry of the underlying periodic lattice. Whereas the shift is arbitrary in coupled Bragg-grating waveguides, the weak refractive in- 
dex contrast imposes the limits on the structure size [5, 23], which may be of the order of centimeters. On the other hand, the system of coupled nanopillar or one-dimensional photonic-crystal waveguides offers the combined advantages of unrestricted dispersion control through non-constrained positioning of individual nanopillars or holes, as illustrated in Fig. 1, and the capability of switching pulses in structures of micrometer dimensions because of the strong field confinement facilitated by the large refractive index contrast. We present the examples of dispersion dependencies in coupled nanopillar and photonic wire waveguides, confirming that fundamentally different band-edge profiles can be obtained by varying just the shift between the identical periodic waveguides.

Whereas different approaches to the control of band-edge dispersion have been discussed in the literature, there is limited research on the pulse shaping under such conditions. We perform finite-difference time-domain (FDTD) simulations of pulse dynamics, and identify novel regimes of spatio-temporal pulse shaping as the input wavelength it tuned close to different spectral regions. In particular, we observe transient spatial switching between the waveguides due to the pulse splitting into slow and regular modes with different spatial pro-

files. We also register asymmetric reshaping of slow-light pulses when the band-edge shape is selected close to quartic profile. We anticipate that such tunable linear dynamics may lead to new possibilities for the frequency conversion processes and all-optical switching [5, 17], since the nonlinear wave mixing is enhanced in the slow-light regime [24, 25].

The paper is organized as follows. In Section 2, we derive analytically the shapes of band-edge dispersion for coupled periodic waveguides. Then, in Section 3 we review the basic properties of coupled nanopillar waveguides and demonstrate the engineering of slowlight dispersion, also presenting analysis of the spatio-temporal dynamics of slow-light pulses in such structures based on FDTD simulations. In Section 4 we demonstrate that similar types of slow-light dispersion can be realized in coupled photonic wire waveguides. Finally, we summarize our key conclusions in Section 5.

\section{Slow-light dispersion in coupled periodic waveguides}

A remarkable property of periodic waveguides is the possibility they afford for reducing the group velocity of optical pulses. The presence of the periodic refractive index modulation along the waveguide leads to Bragg-reflection resonances, and the appearance of photonic band-gaps for a range of frequencies where the light is reflected back [26-28]. In the vicinity 
of the band-edges, the dispersion relation between the optical frequency $(\omega)$ and the Bloch wavenumber $(k)$ is strongly modified, and it is commonly expressed as $\omega \simeq \omega_{b}+D_{2}\left(k-k_{b}\right)^{2}$. Here $\omega_{b}$ and $k_{b}$ denote the values at the band-edge, and $D_{2}$ is the second-order dispersion coefficient. The group velocity $V_{g}=d \omega / d k \simeq \pm 2\left[D_{2}\left(\omega-\omega_{b}\right)\right]^{1 / 2}$ gradually reduces to zero as the frequency is tuned towards the edge of a transmission band.

In this section, we perform the general analysis of the modification of band-edge dispersion in photonic structures consisting of parallel waveguides. We consider two-dimensional modulation of the optical refractive index with the characteristic period $a$ in the propagation direction $(z)$, with the dielectric constant profile written as:

$$
\varepsilon(x, z)=\varepsilon_{p}(x, z)+\varepsilon_{w}(x, z)+\varepsilon_{w}\left(-x+x_{0},-z+z_{0}\right),
$$

where $x$ and $z$ are the transverse and longitudinal coordinates, respectively, $\varepsilon_{w}(x, z)$ describes the profile of a single waveguide, $\left(x_{0}, z_{0}\right)$ is the relative offset of the second waveguide, the function $\varepsilon_{p}(x, z)$ describes the background refractive index. Then, the periodicity condition is expressed as

$$
\varepsilon_{p}(x, z) \equiv \varepsilon_{p}(x, z+a), \quad \varepsilon_{w}(x, z) \equiv \varepsilon_{w}(x, z+a) .
$$

We also require that the individual waveguides have exactly the same profiles, up to the reflection and coordinate shift:

$$
\varepsilon_{p}(x, z) \equiv \varepsilon_{p}\left(-x+x_{0},-z+z_{0}\right)
$$

We note that these conditions are satisfied for a variety of different photonic structures with coupled periodic waveguides, including the following situations:

(i) $\varepsilon_{p}(x, z)=$ const, which is the case for coupled Bragg-grating waveguides [17], arrays of nanopillars [19-22], or coupled one-dimensional photonic-crystal waveguides shown schematically in Fig. 1.

(ii) $\varepsilon_{p}(x, z)$ describes hexagonal photonic-crystal structure, and $x_{0}$ and $z_{0}$ correspond to the basis vectors of the photonic lattice [12].

To be specific, in the following we consider the TM modes (electric field polarized in y-axis), and similar analysis can be performed for TE-polarized waves. Then, the dispersion 
of guided modes can be determined based on the scalar Helmholtz equation for the complex electric field envelopes $E(x, z)$,

$$
\Delta E+\varepsilon(x, z) \omega^{2} c^{-2} E=0
$$

where $\omega$ is the angular frequency and $c$ is the speed of light in vacuum. First, we define the modes of individual waveguides with $\varepsilon_{1}(x, z)=\varepsilon_{p}(x, z)+\varepsilon_{w}(x, z)$ or $\varepsilon_{2}(x, z)=\varepsilon_{p}(x, z)+$ $\varepsilon_{w}\left(-x+x_{0},-z+z_{0}\right)$. Due to the periodicity condition (2) and additional symmetry (3), the waveguides support equivalent sets of modes, which satisfy the Bloch condition:

$$
\begin{aligned}
& E_{1}\left(x, z ; \omega_{1}, k\right)=\psi_{1}\left(x, z ; \omega_{1}, k\right) \exp (i k z), \\
& E_{2}\left(x, z ; \omega_{2}, k\right)=\psi_{2}\left(x, z ; \omega_{2}, k\right) \exp (i k z),
\end{aligned}
$$

where the subscripts 1 and 2 refer to the corresponding waveguides, $\psi$ are the eigenfunctions which are localized in $\mathrm{x}$ and periodic in $z$, and $k$ is the Bloch wave-number. The dispersion dependence $\omega_{1}(k)$ and $\omega_{2}(k)$ can be determined by solving the wave equation (3), using well established approximate analytical or exact numerical methods [26-28]. We note that in dielectric structures $\omega_{j}(k) \equiv \omega_{j}(-k)$ and $\psi_{j}\left(x, z ; \omega_{1}, k\right) \equiv \psi_{j}^{*}\left(x, z ; \omega_{1},-k\right)$, where $*$ stands for complex conjugation. On the other hand, due to the symmetry conditions, $\omega_{1}(k) \equiv \omega_{2}(-k)$, and we can always choose the absolute phase to satisfy $\psi_{2}\left(x, z ; \omega_{1}, k\right) \equiv \psi_{1}\left(-x+x_{0},-z+\right.$ $\left.z_{0} ; \omega_{1},-k\right)$. Then, we conclude that

$$
\begin{aligned}
& \omega_{1}( \pm k) \equiv \omega_{2}( \pm k) \\
& \psi_{2}\left(x, z ; \omega_{1}, k\right) \equiv \psi_{1}^{*}\left(-x+x_{0},-z+z_{0} ; \omega_{1}, k\right) .
\end{aligned}
$$

Now, we seek solutions for the coupled-waveguide structure defined by Eq. (1) as a combination of individual modes with the amplitudes $A_{1}$ and $A_{2}$,

$E(x, z ; \omega, k)=A_{1} E_{1}\left(x, z ; \omega_{1}, k\right)+A_{2} E_{2}\left(x, z ; \omega_{1}, k\right)=\left[A_{1} \psi_{1}\left(x, z ; \omega_{1}, k\right)+A_{2} \psi_{1}^{*}\left(-x+x_{0},-z+z_{0} ; \omega_{1}, k\right)\right] \exp$

We substitute Eq. (7) into Eq. (4), multiply the resulting equation by $E_{1}^{*}$ or $E_{2}^{*}$, and integrate over the area $(-\infty<x<+\infty, 0<z<a)$. Then, we obtain a set of two equations for the amplitudes $A_{1}$ and $A_{2}$, which have a non-trivial solution when the following condition is satisfied:

$$
\left(\delta b_{1}+\omega_{1}^{2} b_{2}\right)^{2}=\left|\delta b_{3}+\omega_{1}^{2} b_{4}\right|^{2}
$$


Here $\delta=\omega^{2}-\omega_{1}^{2}$ defines the frequency detuning due to waveguide coupling, and the coupling coefficients are:

$$
\begin{aligned}
& b_{1}=\left\langle\left[\varepsilon_{p}(x, z)+\varepsilon_{w}(x, z)+\varepsilon_{w}\left(-x+x_{0},-z+z_{0}\right)\right]\left|\psi_{1}\left(x, z ; \omega_{1}, k\right)\right|^{2}\right\rangle, \\
& b_{2}=\left\langle\varepsilon_{w}\left(-x+x_{0},-z+z_{0}\right)\left|\psi_{1}\left(x, z ; \omega_{1}, k\right)\right|^{2}\right\rangle, \\
& b_{3}=\left\langle\left[\varepsilon_{p}(x, z)+\varepsilon_{w}(x, z)+\varepsilon_{w}\left(-x+x_{0},-z+z_{0}\right)\right] \psi_{1}\left(x, z ; \omega_{1}, k\right) \psi_{1}\left(-x+x_{0},-z+z_{0} ; \omega_{1}, k\right)\right\rangle, \\
& b_{4}=\left\langle\varepsilon_{w}\left(-x+x_{0},-z+z_{0}\right) \psi_{1}\left(x, z ; \omega_{1}, k\right) \psi_{1}\left(-x+x_{0},-z+z_{0} ; \omega_{1}, k\right)\right\rangle,
\end{aligned}
$$

where the brackets $<\bullet>$ denote $\int_{-\infty}^{+\infty} d x \int_{0}^{a} d z(\bullet)$.

We consider the case of weakly coupled waveguides. Then, we estimate that $b_{1} \simeq O(1)$, $b_{2,3,4} \simeq O(\xi)$, and $\delta \simeq O(\xi)$, where $\xi$ is a small parameter. By keeping the lowest-order terms in Eq. (8), we obtain the following asymptotic relation

$$
\left(\delta b_{1}+\omega_{1}^{2} b_{2}\right)^{2} \simeq \omega_{1}^{4}\left|b_{4}\right|^{2}
$$

and determine the dispersion relation analytically:

$$
\delta=\omega^{2}-\omega_{1}^{2} \simeq \omega_{1}^{2}\left(-b_{2} / b_{1} \pm\left|b_{4}\right| / b_{1}\right)
$$

We assume that the individual waveguides have the commonly occurring parabolic shape of the dispersion curves at the edge of the Brillouin zone,

$$
\omega_{1}(k) \simeq \omega_{1 b}+D_{2}\left(k-k_{1 b}\right)^{2}
$$

where $k_{1 b}=\pi / a$. We introduce the notation $\kappa=k-k_{1 b}$, and expand the coupling coefficients in $\kappa$,

$$
\begin{aligned}
& b_{1} \simeq b_{1 b}+b_{1 b 2} \kappa^{2}, \\
& b_{2} \simeq b_{2 b}+b_{2 b 2} \kappa^{2}, \\
& b_{4} \simeq b_{4 b}+i b_{4 b 1} \kappa+b_{4 b 2} \kappa^{2} .
\end{aligned}
$$

We substitute Eqs. (12) and (13) into Eq. (11), take into account that $\omega^{2}-\omega_{1}^{2} \simeq 2(\omega-$ $\left.\omega_{1}\right) \omega_{1}$, and obtain the final expression for mode dispersion in coupled waveguides close to the band-edge, in the regime of slow-light propagation:

$$
\omega \simeq\left[\omega_{1 b}+D_{2} \kappa^{2}\right]\left\{1-\frac{b_{2 b}+b_{2 b 2} \kappa^{2} \pm\left[\left(b_{4 b}+b_{4 b 2} \kappa^{2}\right)^{2}+\kappa^{2} b_{4 b 1}^{2}\right]^{1 / 2}}{2\left(b_{1 b}+b_{1 b 2} \kappa^{2}\right)}\right\} .
$$


We now analyze particular cases, considering symmetric waveguides with $\varepsilon_{p, w}(x, z) \equiv$ $\varepsilon_{p, w}(-x, z) \equiv \varepsilon_{p, w}(x,-z)$.

(i) If there is no shift between the waveguides, $z_{0}=0$, then $b_{4 b 1}=0$, and

$$
\omega \simeq\left[\omega_{1 b}+D_{2} \kappa^{2}\right]\left\{1-\frac{b_{2 b}+b_{2 b 2} \kappa^{2} \pm\left[b_{4 b}+b_{4 b 2} \kappa^{2}\right]}{2\left(b_{1 b}+b_{1 b 2} \kappa^{2}\right)}\right\} .
$$

In this case, the dispersion branches are split vertically, as illustrated in Fig. 2(b). At the band-edge, there appears a single slow-light mode, featuring conventional quadratic dispersion dependence.

(ii) If the waveguides are shifted by half of the modulation period, $z_{0}=a / 2$, then $b_{2 b}=$ $b_{4 b}=0$, and

$$
\omega \simeq\left[\omega_{1 b}+D_{2} \kappa^{2}\right]\left\{1-\frac{b_{2 b 2} \kappa^{2} \pm\left|\kappa b_{4 b 1}\right|}{2\left(b_{1 b}+b_{1 b 2} \kappa^{2}\right)}\right\} .
$$

In this case, the dispersion branches predominantly split horizontally, as illustrated in Fig. 2(c). Accordingly, there appears the so-called split band-edge, associated with the co-existence of two distinct slow-light modes in the same frequency region as the photonic band-edge.

(iii) By choosing the intermediate shifts between the waveguides, it becomes possible to tailor the dispersion dependencies, as they are transformed between the cases (i) and (ii). We elaborate more on this possibility in the following Section.

\section{Slow-light dynamics in dispersion-engineered coupled nanopillar waveguides}

We now demonstrate how the band-edge dispersion can be engineered in coupled nanopillar waveguides, shown schematically in Fig. 1(a). The potential for practical realization of nanopillar waveguide structures was demonstrated in experimental studies [29-32]. For the numerical simulations presented below, we solve Maxwell's equations in a two-dimensional geometry for TM-polarized electromagnetic waves (with the electric field polarized along $y$ axis). We choose the nanopillar diameter $0.29 a$, the material refractive index 2.5 , and the transverse separation between the coupled waveguides $x_{0}=a$. For convenience, we normalize the time to $\tau=a / c$, Bloch wavenumber to $2 \pi / a$, and the frequency is measured in multiples of $a / \lambda$, where $\lambda$ is the wavelength in vacuum. In FDTD simulations, we use

pulses with the full-width at half-maximum of the frequency spectrum equal to $0.5 \%$ of the central frequency. We estimate that, in order to observe the slow-light propagation at 
$1.55 \mu \mathrm{m}$, one can use the structure with the period $a \simeq 0.62 \mu \mathrm{m}$, the nanopillar diameter of $180 \mathrm{~nm}$, the time scale $\tau \simeq 2.07 \mathrm{fs}$, and the pulse duration of $655 \mathrm{fs}$.

\section{A. Symmetrically coupled waveguides with no longitudinal shift}

We start by considering the case of zero longitudinal offset $\left(z_{0}=0\right)$, when the photonic structure is symmetric as shown in Fig. 3(a). Calculations of the dispersion dependencies were performed using the freely available MIT Photonic Bands (MPB) software package utilizing the plane-wave expansion method [33]. In agreement with the general analysis presented in Sec. 2, the dispersion branches are split vertically, see Fig. 3(b). Accordingly, the slow-light regime is realized for both modes at the edge of the Brillouin zone, corresponding to the same value of the normalized wavenumber $(k=0.5)$, see Fig. 3(b). We note that the first mode has an odd symmetry [see Fig. 3(d)], such that the electric field has a $\pi$ phase shift between the waveguides. On the other hand, the second modes the has an even symmetry [see Fig. 3(e)], and the coupled waveguides are in-phase. This situation is similar to the mode structure in a conventional directional coupler [34-36], indicating the possibility of light switching between the parallel waveguides. However, the mode cut-offs and the associated reduction of their group velocities appear at different frequencies, and we perform FDTD simulation to investigate the pulse dynamics in the system.

As a characteristic example demonstrating the effect of beating between the modes on the pulse dynamics, we tune the pulse frequency close to the cut-off of the second mode. The pulse spectrum is shown with grey-shaded stripe in Fig. 3(b), at the frequency around $\omega \simeq$ 0.3784. We see that, in this frequency region, both modes can propagate in the structure. However, the two modes have significantly different group velocities, since the slow-light propagation is realized only for the second mode. Indeed, our simulations demonstrate that the leading edge of the pulse has an odd symmetry, associated with the faster-propagating first mode, see Fig. 4(a). At a later time [Fig. 4(b)], periodic switching of light is observed along the waveguides, due to the transient overlap between the slowly propagating even mode and the odd-mode state. Finally, at longer times only the slow even mode remains inside the structure [Fig. 4(c)], and the spatial switching again disappears.

\section{B. Coupled waveguides shifted by half a period}

We now consider the light dynamics in the case when the waveguides are shifted longitudinally by half of the period, as shown in Fig. 5(a). In agreement with the general predictions 
of Sec. 2, now the dispersion curves split horizontally [21], resulting in the appearance of the split band-edge, see Fig. 5(b). In this case, there appear two band edges with different wave-numbers, equally separated from the Brillouin zone edge. However, the cut-off frequencies for the two modes now coincide. In agreement with the previous results for other types of coupled periodic waveguides $[12,17]$, we observe that this type of dispersion also facilitates switching of slow-light pulses in coupled nanopillar waveguides. Although the modal profiles are now neither even nor odd [see Figs. 5(d) and (e)], their beating still results in the periodic tunneling of light between the waveguides, as confirmed by numerical simulations presented in Fig. 6. We see that the pulse follows a sinusoidal trajectory, as it periodically switches between the waveguides during the propagation along the structure. Such characteristic pulse trajectory is preserved at all time steps, and this is only possible because both modes propagate in the slow-light regime with the same group velocities.

\section{Coupled waveguides with intermediate longitudinal shift}

Finally, we consider the case of intermediate longitudinal shifts between the coupled periodic waveguides. By continuously varying this structural parameter, we can flexibly control the band-edge dispersion. In particular, for the structure shown in Fig. 7(a), we realize the situation when the first mode dispersion becomes almost flat close to the band-edge, see Fig. 7(b). As a result, there appear two zero group velocity regions at a frequencies in the vicinity of the band edge, see Figs. 7(c). Since the coupled waveguide structure is no longer

symmetric or antisymmetric, the modes feature skewed amplitude and phase profiles, see Figs. 7(d) and (e).

We simulate the pulse dynamics when its central frequency is tuned right at the bandedge $(\omega \simeq 0.4029)$, as marked with the grey-shaded stripe in Fig. 7(b). This frequency is above the cut-off of the second mode, however we observe the periodic light tunneling between the waveguides which is characteristic of two-mode beating, see Fig. 8. Indeed, for frequencies very close to the band-edge, there exist two sections of the dispersion curve above and below the Brillouin zone edge, where the group velocities for the first mode are small and positive. It is the beating between these states that results in the tunneling of light between the waveguides. In contrast to the case of longitudinal shift by half a period discussed above in Sec. $3 \mathrm{~B}$, the switching between the waveguides is not symmetric. Indeed, the switching lengths from the upper to the lower waveguides and back are different, due to the asymmetry of the underlying photonic structure. Nevertheless, we observe that the 
switching is almost complete at certain propagation distances. Therefore, such asymmetric structures can operate as slow-light couplers, with the possibility for fine-tuning the bandedge dispersion.

\section{Photonic wire waveguides}

We now show that the band-edge dispersion can also be controlled in other type of periodic structures, considering the case of coupled photonic wire waveguides shown schematically in Fig. 1(b). Such waveguides have been extensively investigated in recent years, and we choose the parameters based on the characteristics of previously fabricated structures [37, 38]. Specifically, we solve Maxwell's equations in a two-dimensional geometry for TE-polarized electromagnetic waves (with the magnetic field polarized along $y$ axis). We choose the width of individual waveguides equal to the structure period $a$, the hole diameter $0.4 a$, the effective material refractive index 2.798, and the transverse separation between the coupled waveguide centers $x_{0}=1.2 \mathrm{a}$. Similar to the results presented for coupled nanopillar waveguides in Sec. 3, we normalize Bloch wavenumber to $2 \pi / a$, and the frequency is measured in multiples of $a / \lambda$, where $\lambda$ is the wavelength in vacuum. According to the results of Ref. [37, 38], the slow-light propagation at $1.5 \mu \mathrm{m}$ can be realized when the period is of the order of $a \simeq 0.35 \mu \mathrm{m}$.

Whereas the analytical analysis in Sec. 2 is presented for TM-polarized waves, numerical simulations indicate that the dispersion of TE-polarized modes in coupled periodic waveguides follows the same general trends as sketched in Figs. 2(a-c). Specifically, we find that the slow-light regime appears at separate frequency cut-offs for even and odd modes when the holes in adjacent waveguides are aligned (Fig. 9). Then, quartic band-edge dispersion can be realized for a particular value of the longitudinal shift between the holes, see Fig. 10. Finally, the split band-edge is observed when the holes are shifted by half a period, see Fig. 11. Due to the similarity of dispersion characteristics and the symmetries of mode profiles with those of nanopillar waveguides, we expect that the pulse propagation in photonic wires would mimic closely the key features of spatial-temporal dynamics discussed in Sec. 3.

\section{Conclusions}

We have shown that dispersion characteristics of slow-light at photonic band-edges can be flexibly controlled in coupled periodic waveguides, simply by changing the longitudinal offset. Our general theoretical analysis provides a link between the variety of previously considered 
coupled guiding structures, formulating the generic basis for dispersion engineering. We further demonstrate how various dispersion dependencies can be realized for a system of coupled nanopillar waveguides comprising periodic rows of dielectric rods and photonic wire waveguides with a periodic set of holes. In such structures, the longitudinal offset between the rods or holes can be freely varied, offering full flexibility in tailoring the dispersion properties. Our numerical FDTD simulations illustrate distinctly different regimes of spatiotemporal pulse dynamics, demonstrating nontrivial dependencies on the frequency detuning from the band-edge and high sensitivity to the structure symmetry.

These results suggest several directions for further studies. We note that by optimizing the value of the longitudinal shift between the periodic waveguides, it is possible to obtain quartic band-edge dispersion. It was predicted that such flat band edges can lead to an increased density of states at frequencies near the band edge $[9,10]$, and this may offer a potential to enhance the characteristics of slow-light resonators. Another parameter which needs to be carefully addressed is a distance between photonic wires or transverse offset between two rows of nanopillars providing the optimal coupling strength for effective interactions. The demonstrated control over the phase velocities and spatial profiles of slow-light modes also suggest the potential for flexible engineering of wave-mixing processes and all-optical switching with discrete solitons [39], taking advantage of nonlinearity enhancement in the slow-light regime.

\section{Acknowledgements}

We thank M. de Sterke, L. Botten, K. Dossou, C. Monat, and B. J. Eggleton for useful discussions and comments.

This work was supported by the Australian Research Council under the Centres of Excellence Program, and through the Australian Partnership for Advanced Computing National Facility (APAC). Part of this project was completed during the visit of A.A.S. to McMaster University (Canada) supported by the Australian Academy of Science travel grant. D.N.C. acknowledges partial support by the Deutsche Forschungsgemeinschaft through the project FOR 557. A.V.L. acknowledges partial support from European Commission FP6, project NewTon, NMP4-CT-2005-017160. 


\section{References}

1. X. Letartre, C. Seassal, C. Grillet, P. Rojo Romeo, P. Viktorovitch, M. L. d'Yerville, D. Cassagne, and C. Jouanin, "Group velocity and propagation losses measurement in a singleline photonic-crystal waveguide on InP membranes," Appl. Phys. Lett. 79, 2312-2314 (2001).

2. Y. A. Vlasov, M. O'Boyle, H. F. Hamann, and S. J. McNab, "Active control of slow light on a chip with photonic crystal waveguides," Nature 438, 65-69 (2005).

3. H. Gersen, T. J. Karle, R. J. P. Engelen, W. Bogaerts, J. P. Korterik, N. F. Hulst, van, T. F. Krauss, and L. Kuipers, "Real-space observation of ultraslow light in photonic crystal waveguides," Phys. Rev. Lett. 94, 073903-4 (2005).

4. R. S. Jacobsen, A. V. Lavrinenko, L. H. Frandsen, C. Peucheret, B. Zsigri, G. Moulin, J. Fage Pedersen, and P. I. Borel, "Direct experimental and numerical determination of extremely high group indices in photonic crystal waveguides," Opt. Express 13, 7861-7871 (2005).

5. J. T. Mok, C. M. de Sterke, I. C. M. Littler, and B. J. Eggleton, "Dispersionless slow light using gap solitons," Nature Physics 2, 775-780 (2006).

6. M. D. Settle, R. J. P. Engelen, M. Salib, A. Michaeli, L. Kuipers, and T. F. Krauss, "Flatband slow light in photonic crystals featuring spatial pulse compression and terahertz bandwidth," Opt. Express 15, 219-226 (2007).

7. S. C. Huang, M. Kato, E. Kuramochi, C. P. Lee, and M. Notomi, "Time-domain and spectraldomain investigation of inflection-point slow-light modes in photonic crystal coupled waveguides," Opt. Express 15, 3543-3549 (2007).

8. R. J. P. Engelen, Y. Sugimoto, Y. Watanabe, J. P. Korterik, N. Ikeda, N. F. Hulst, van, K. Asakawa, and L. Kuipers, "The effect of higher-order dispersion on slow light propagation in photonic crystal waveguides," Opt. Express 14, 1658-1672 (2006).

9. A. Figotin and I. Vitebskiy, "Gigantic transmission band-edge resonance in periodic stacks of anisotropic layers," Phys. Rev. E 72, 036619-12 (2005).

10. M. Ibanescu, S. G. Johnson, D. Roundy, C. Luo, Y. Fink, and J. D. Joannopoulos, "Anomalous dispersion relations by symmetry breaking in axially uniform waveguides," Phys. Rev. Lett. 92, 063903-4 (2004).

11. A. A. Sukhorukov, C. J. Handmer, C. M. de Sterke, and M. J. Steel, "Slow light with flat or offset band edges in few-mode fiber with two gratings," Opt. Express 15, 17954-17959 
(2007).

12. S. W. Ha, A. A. Sukhorukov, K. B. Dossou, L. C. Botten, A. V. Lavrinenko, D. N. Chigrin, and Yu. S. Kivshar, "Dispersionless tunneling of slow light in antisymmetric photonic crystal couplers," Opt. Express 16, 1104-1114 (2008).

13. R. Marz and H. P. Nolting, "Spectral properties of asymmetrical optical directional-couplers with periodic structures," Opt. Quantum Electron. 19, 273-287 (1987).

14. G. Perrone, M. Laurenzano, and I. Montrosset, "Design and feasibility analysis of an innovative integrated grating-assisted add-drop multiplexer," J. Lightwave Technol. 19, 1943-1948 (2001).

15. M. Aslund, J. Canning, L. Poladian, C. M. de Sterke, and A. Judge, "Antisymmetric grating coupler: experimental results," Appl. Optics 42, 6578-6583 (2003).

16. J. M. Castro, D. F. Geraghty, S. Honkanen, C. M. Greiner, D. Iazikov, and T. W. Mossberg, "Demonstration of mode conversion using anti-symmetric waveguide Bragg gratings," Opt. Express 13, 4180-4184 (2005).

17. S. Ha, A. A. Sukhorukov, and Yu. S. Kivshar, "Slow-light switching in nonlinear Bragg-grating couplers," Opt. Lett. 32, 1429-1431 (2007).

18. H. Benisty, "Modal analysis of optical guides with two-dimensional photonic band-gap boundaries," J. Appl. Phys. 79, 7483-7492 (1996).

19. D. N. Chigrin, A. V. Lavrinenko, and C. M. S. Torres, "Nanopillars photonic crystal waveguides," Opt. Express 12, 617-622 (2004).

20. D. N. Chigrin, A. V. Lavrinenko, and C. M. S. Torres, "Numerical characterization of nanopillar photonic crystal waveguides and directional couplers," Opt. Quantum Electron. 37, 331$341(2005)$.

21. D. N. Chigrin, S. V. Zhukovsky, A. V. Lavrinenko, and J. Kroha, "Coupled nanopillar waveguides optical properties and applications," Phys. Status Solidi A 204, 3647-3661 (2007).

22. Y. G. Boucher, A. V. Lavrinenko, and D. N. Chigrin, "Out-of-phase coupled periodic waveguides: a "couplonic" approach," Opt. Quantum Electron. 39, 837-847 (2007).

23. J. T. Mok, M. Ibsen, C. M. de Sterke, and B. J. Eggleton, "Dispersionless slow light with 5-pulse-width delay in fibre Bragg grating," Electron. Lett. 43, 1418-1419 (2007).

24. M. Soljacic and J. D. Joannopoulos, "Enhancement of nonlinear effects using photonic crystals," Nat. Mater. 3, 211-219 (2004). 
25. R. S. Jacobsen, K. N. Andersen, P. I. Borel, J. Fage Pedersen, L. H. Frandsen, O. Hansen, M. Kristensen, A. V. Lavrinenko, G. Moulin, H. Ou, C. Peucheret, B. Zsigri, and A. Bjarklev, "Strained silicon as a new electro-optic material," Nature 441, 199-202 (2006).

26. P. Yeh, Optical Waves in Layered Media (John Wiley \& Sons, New York, 1988).

27. J. D. Joannopoulos, R. D. Meade, and J. N. Winn, Photonic Crystals: Molding the Flow of Light (Princeton University Press, Princeton, 1995).

28. P. St. J. Russell, T. A. Birks, and F. D. Lloyd Lucas, "Photonic Bloch waves and photonic band gaps," in Confined Electrons and Photons, E. Burstein and C. Weisbuch, eds., (Plenum, New York, 1995), pp. 585-633.

29. M. Tokushima, H. Yamada, and Y. Arakawa, "1.5- $\mu \mathrm{m}$-wavelength light guiding in waveguides in square-lattice-of-rod photonic crystal slab," Appl. Phys. Lett. 84, 4298-4300 (2004).

30. S. Assefa, P. T. Rakich, P. Bienstman, S. G. Johnson, G. S. Petrich, J. D. Joannopoulos, L. A. Kolodziejski, E. P. Ippen, and H. I. Smith, "Guiding $1.5 \mu \mathrm{m}$ light in photonic crystals based on dielectric rods," Appl. Phys. Lett. 85, 6110-6112 (2004).

31. X. Y. Ao, L. Liu, L. Wosinski, and S. L. He, "Polarization beam splitter based on a twodimensional photonic crystal of pillar type," Appl. Phys. Lett. 89, 171115-3 (2006).

32. J. She, E. Forsberg, X. Y. Ao, and S. L. He, "High-efficiency polarization beam splitters based on a two-dimensional polymer photonic crystal," J. Opt. A-Pure Appl. Opt. 8, 345349 (2006).

33. S. G. Johnson and J. D. Joannopoulos, "Block-iterative frequency-domain methods for Maxwell's equations in a planewave basis," Opt. Express 8, 173-190 (2001).

34. S. M. Jensen, "The nonlinear coherent coupler," IEEE Trans. Microw. Theory Tech. MTT30, 1568-1571 (1982).

35. A. A. Maier, "Optical transistors and bistable elements on the basis of non-linear transmission of light by the systems with unidirectional coupled waves," Kvantov. Elektron. 9, 2296-2302 (1982) (in Russian) [English translation: Quantum Electron. 12, 1490-1494 (1982)].

36. S. R. Friberg, Y. Silberberg, M. K. Oliver, M. J. Andrejco, M. A. Saifi, and P. W. Smith, "Ultrafast all-optical switching in a dual-core fiber nonlinear coupler," Appl. Phys. Lett. 51, 1135-1137 (1987).

37. D. Goldring, U. Levy, and D. Mendlovic, "Highly dispersive micro-ring resonator based on one dimensional photonic crystal waveguide design and analysis," Opt. Express 15, 3156-3168 
(2007).

38. D. Goldring, U. Levy, I. E. Dotan, A. Tsukernik, M. Oksman, I. Rubin, Y. David, and D. Mendlovic, "Experimental measurement of quality factor enhancement using slow light modes in one dimensional photonic crystal," Opt. Express 16, 5585-5595 (2008).

39. N. K. Efremidis and D. N. Christodoulides, "Discrete solitons in nonlinear zigzag optical waveguide arrays with tailored diffraction properties," Phys. Rev. E 65, 056607-11 (2002). 


\section{FIGURE CAPTIONS}

Fig. 1. Sketch of coupled periodic waveguides, consisting of (a) two arrays of nanopillars or (b) two arrays of holes in coupled photonic wire waveguides.

Fig. 2. Characteristic dispersion dependencies (top row) and the corresponding group velocities (bottom row) close to the band-edge for (a) Uncoupled period waveguides; (b) Coupled periodic waveguides with no longitudinal offset $\left(z_{0}=0\right)$, and (c) Coupled waveguides with the longitudinal offset by half a period $\left(z_{0}=a / 2\right)$.

Fig. 3. (a) Transverse refractive index profile of coupled nanopillar waveguides, with zero longitudinal offset between the lower and upper rows of nanopillars $\left(z_{0}=0\right)$. (b) Dispersion dependencies of the normalized frequency on the Bloch wavenumbers for the two fundamental guided modes, shown with solid (mode 1) and dashed (mode 2) lines, respectively. Grey shading indicates the pulse spectrum used in FDTD numerical simulations. (c) Dependence of the normalized group velocity of the guided modes on the Bloch wave-number. $(\mathrm{d}, \mathrm{e})$ Characteristic amplitude and phase profiles of the modes, corresponding to the marked points in plots (b) and (c) with the Bloch wave-number $k=0.475$.

Fig. 4. Pulse dynamics in the coupled nanopilar waveguides shown in Fig. 3(a). The central pulse frequency is $\omega \simeq 0.3784$, and the pulse spectrum truncated at full-width of half-maximum is shown with the grey shading at the corresponding frequency region in Fig. 3(b). Shown are the snapshots of the electric field component $E_{z}$ at different times, as indicated by labels.

Fig. 5. Dispersion and modal characteristics for coupled waveguides, where the lower and upper rows of nanopillars are shifted by half of the longitudinal periods $\left(z_{0}=0.5 a\right)$. Notations are the same as in Fig. 3, and the mode profiles in (d,e) are shown for $k=0.455$. 
Fig. 6. Pulse dynamics in the coupled nanopilar waveguides shown in Fig. 5(a). The central pulse frequency is $\omega \simeq 0.4035$, and the pulse spectrum truncated at full-width of halfmaximum is shown with the grey shading in Fig. 5(b). Shown are the snapshots of the electric field component $E_{z}$ at different times, as indicated by labels.

Fig. 7. Dispersion and modal characteristics for coupled waveguides, where the lower and upper rows of nanopillars are shifted by $z_{0}=0.35 a$. Notations are the same as in Fig. 3, and the mode profiles in $(\mathrm{d}, \mathrm{e})$ are shown for $k=0.475$.

Fig. 8. Pulse dynamics in the coupled nanopilar waveguides shown in Fig. 7(a). The central pulse frequency is $\omega \simeq 0.4029$, and the pulse spectrum truncated at full-width of half-maximum is marked with the grey shading at the corresponding frequency region in Fig. 7(b). Shown are the snapshots of the electric field component $E_{z}$ at different times, as indicated by labels.

Fig. 9. Dispersion and modal characteristics for coupled photonic wire waveguides with zero longitudinal offset between the lower and upper rows of holes $\left(z_{0}=0\right)$. (a) Transverse refractive index profile. (b) Dispersion dependencies of the normalized frequency on the Bloch wavenumbers for the two fundamental guided modes, shown with solid (mode 1) and dashed (mode 2) lines, respectively. (c) Dependence of the normalized group velocity of the guided modes on the Bloch wave-number. (d,e) Characteristic amplitude and phase profiles of the modes, corresponding to the marked points in plots (b) and (c) with the Bloch wave-number $k=0.475$.

Fig. 10. Dispersion and modal characteristics for coupled photonic wire waveguides, where the lower and upper rows of holes are shifted by $z_{0}=0.28 a$. Notations are the same as in Fig. 9.

Fig. 11. Dispersion and modal characteristics for coupled photonic wire waveguides, where the lower and upper rows of holes are shifted by half of the longitudinal periods $\left(z_{0}=0.5 a\right)$. Notations are the same as in Fig. 9. 

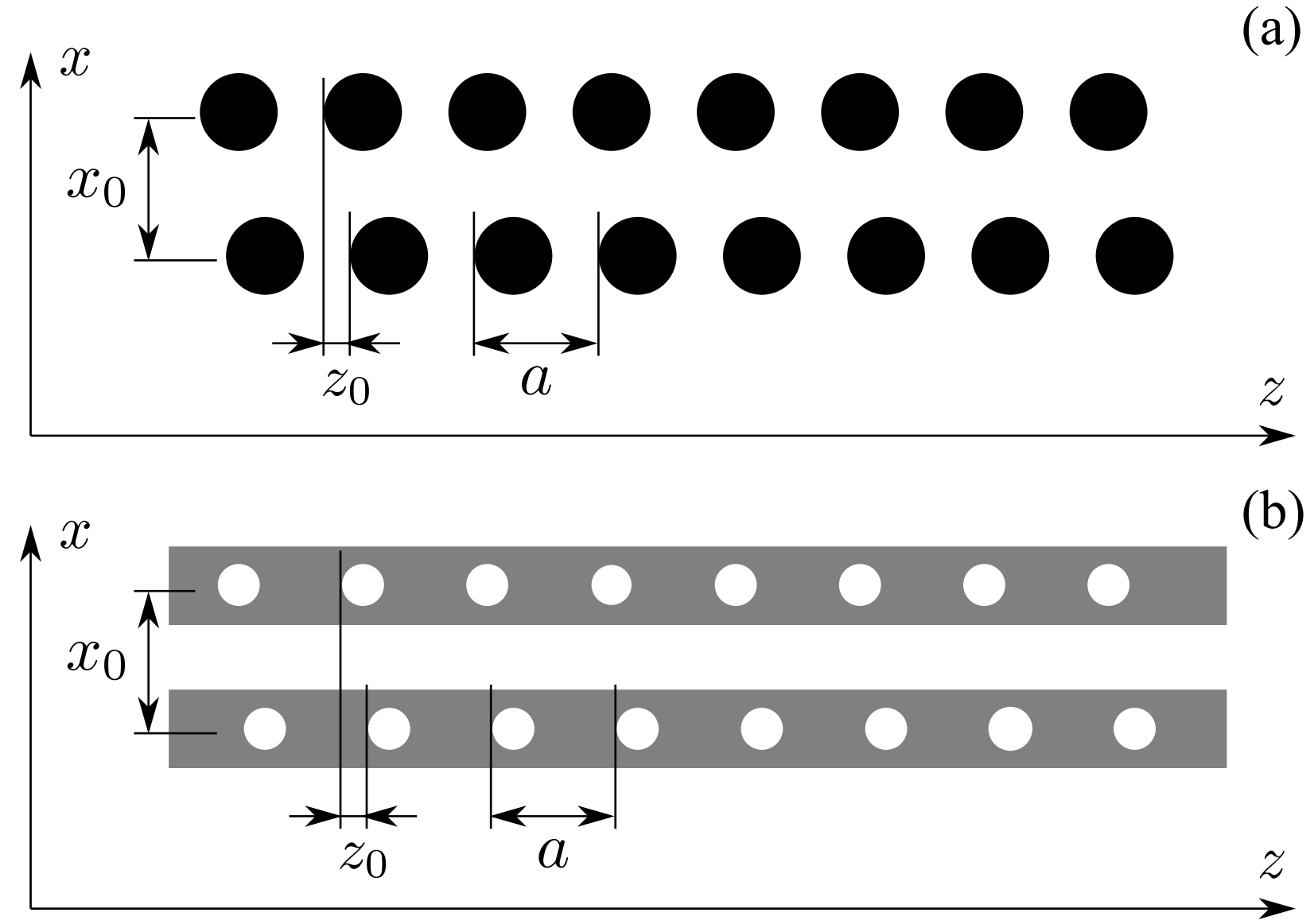

Fig. 1. 
Uncoupled periodic waveguides

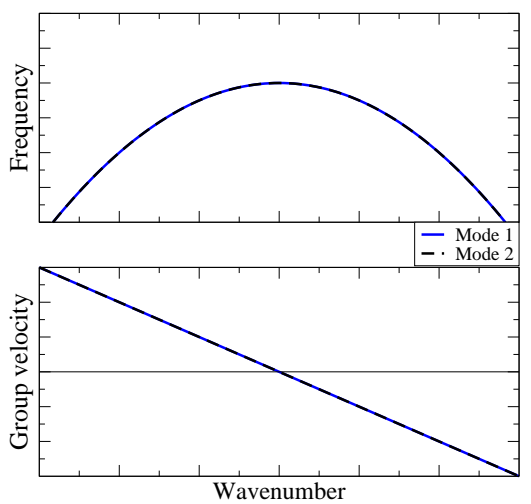

(a)
Coupled; no offset
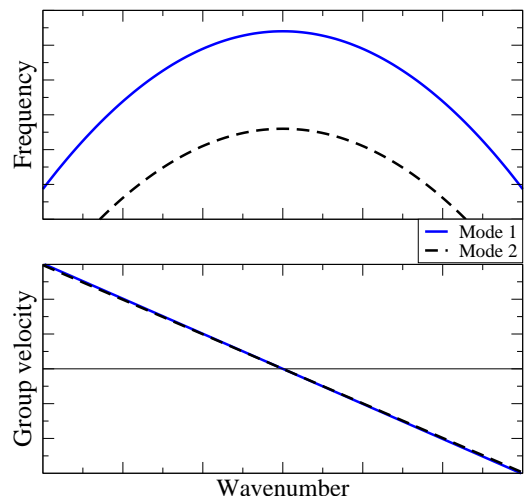

(b)

\section{Coupled; half-period offset}

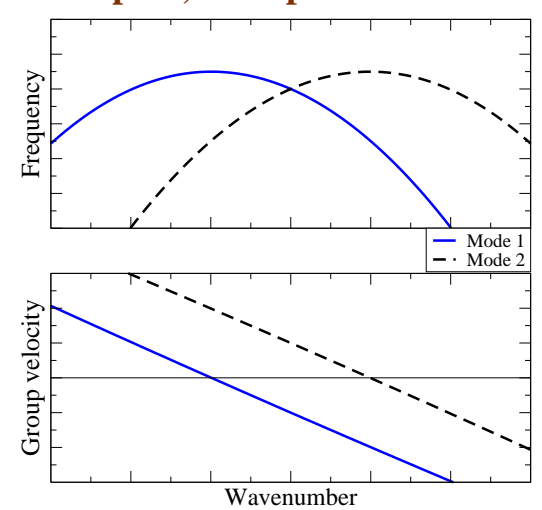

(c)

Fig. 2. 


\section{Rods offset 0}

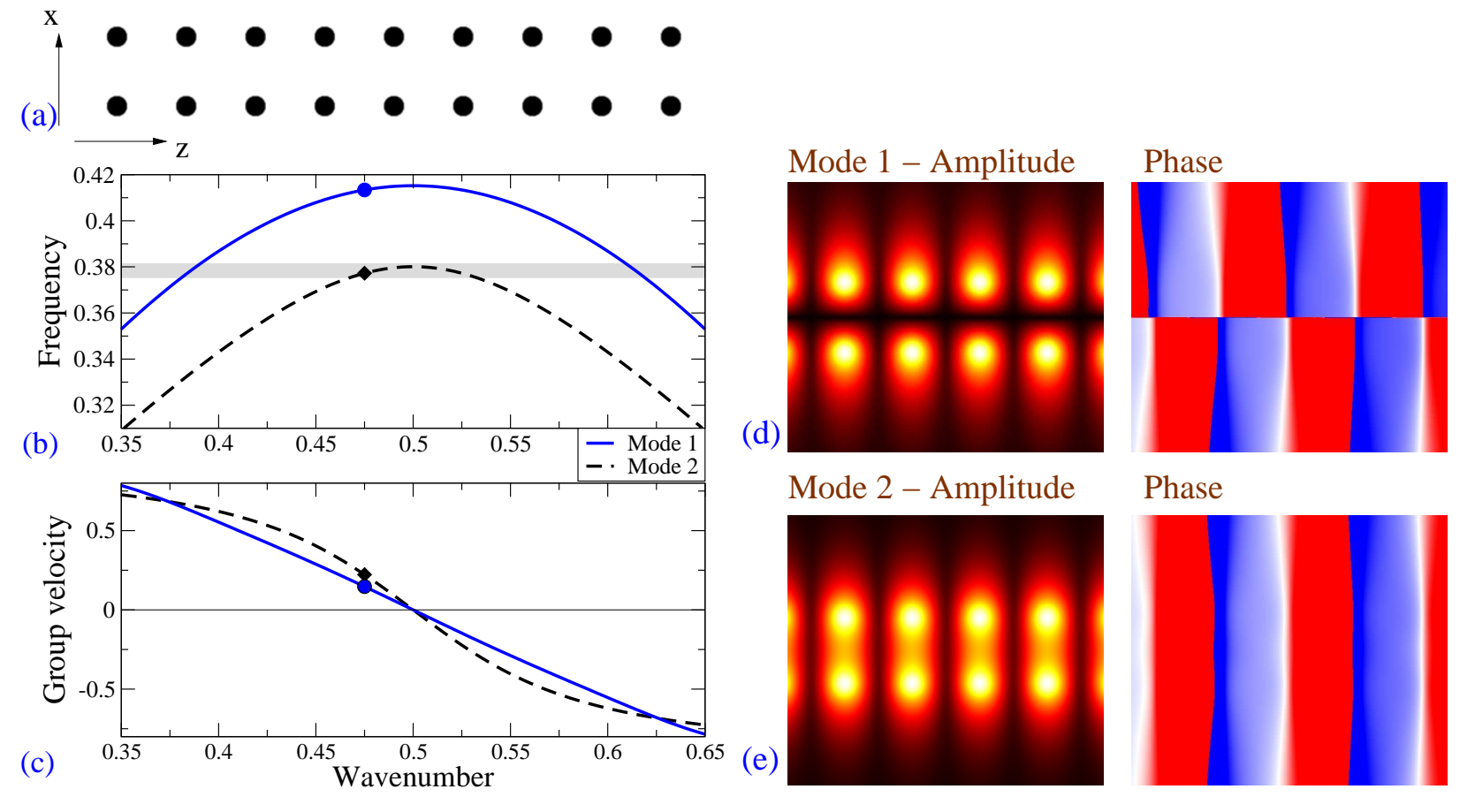

Fig. 3. 
Rods offset 0; Normalized frequency 0.3784

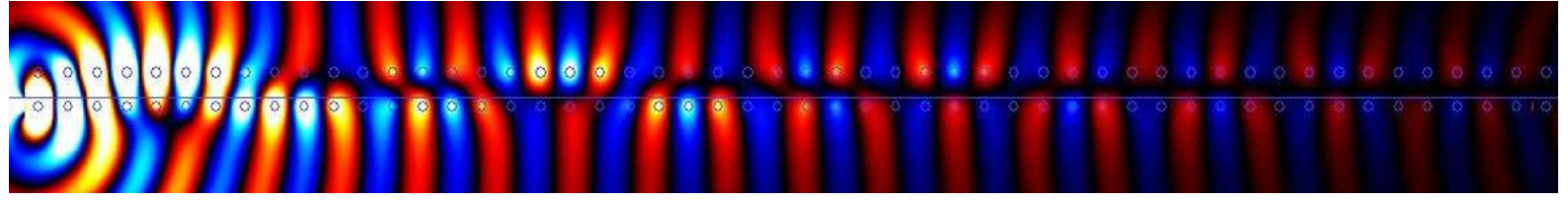

(a) Time $=124$

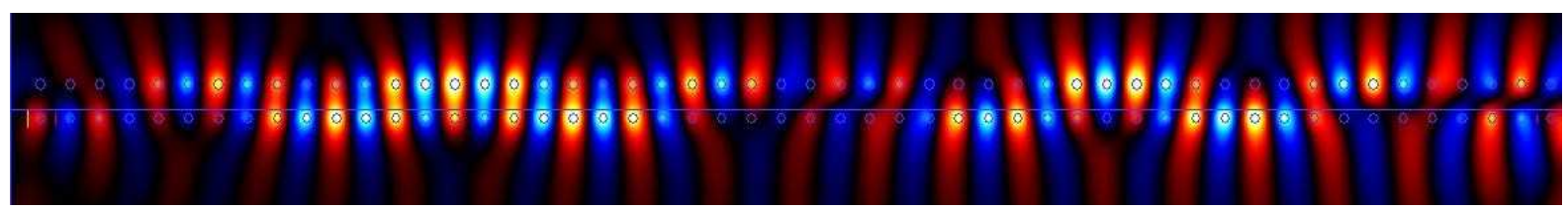

(b) Time $=294$

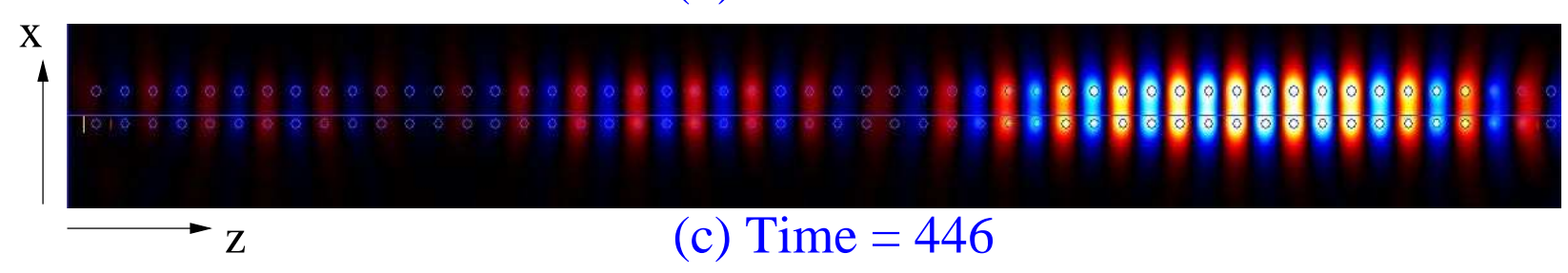

Fig. 4. 
Rods offset 0.5

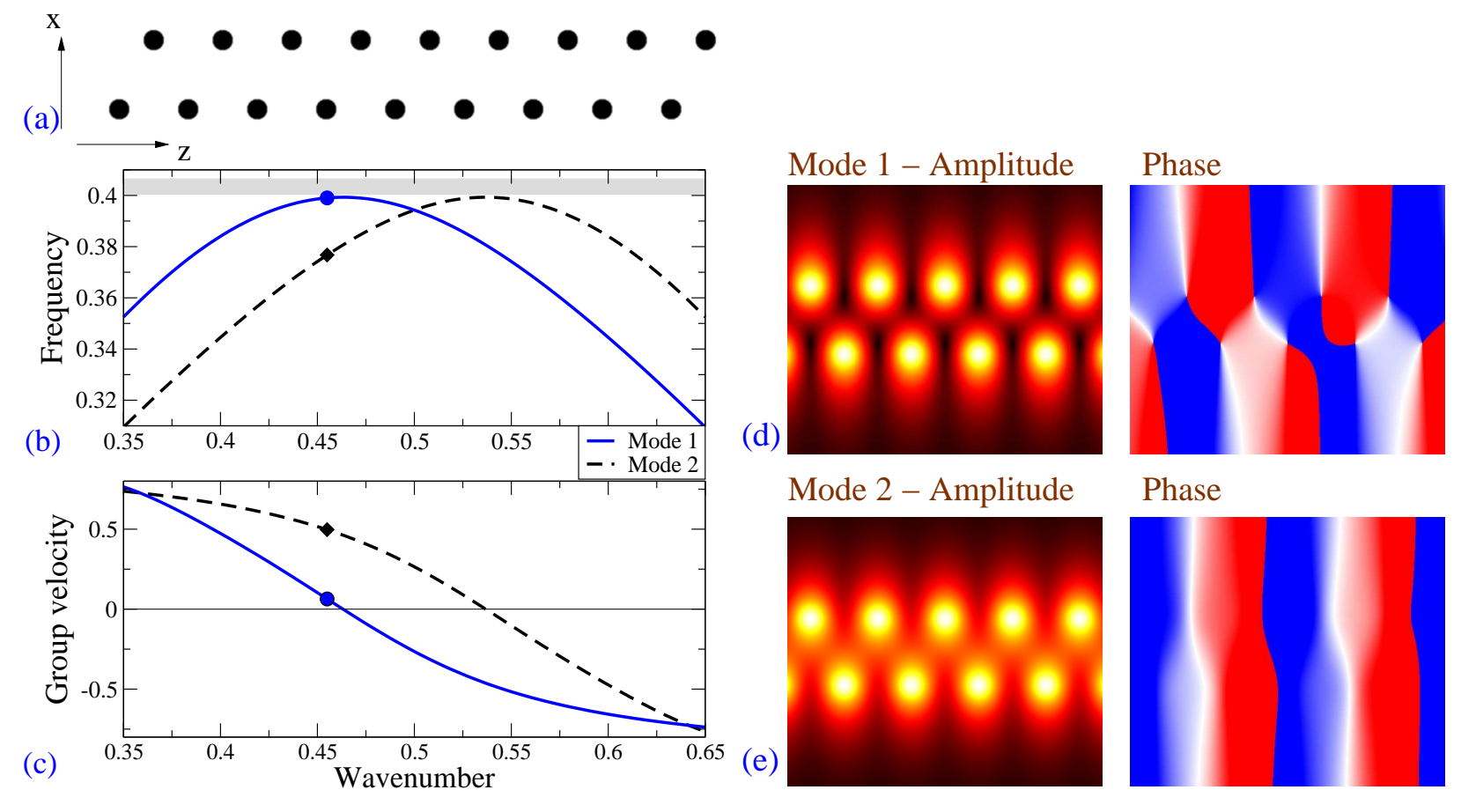

Fig. 5. 
Rods offset 0.5 ; Normalized frequency 0.4035

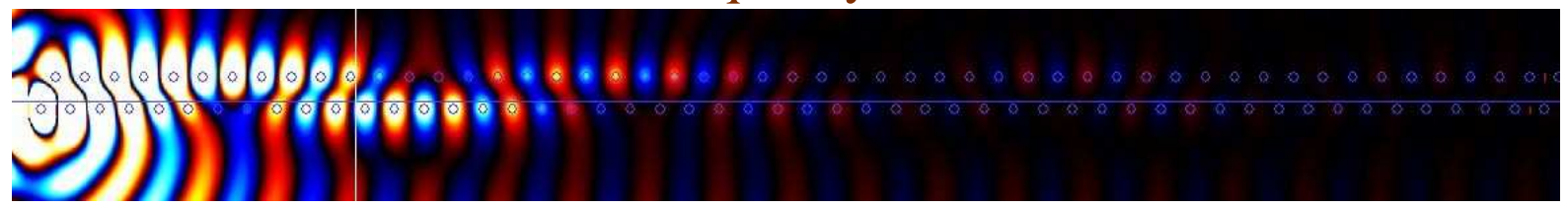

(a) Time $=134$

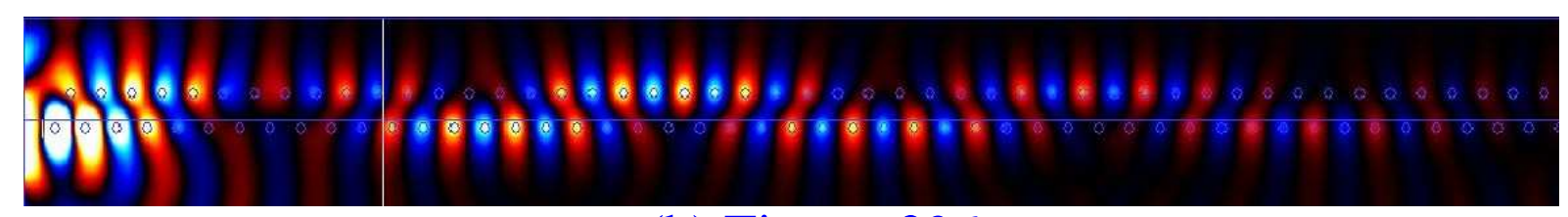

(b) Time $=296$

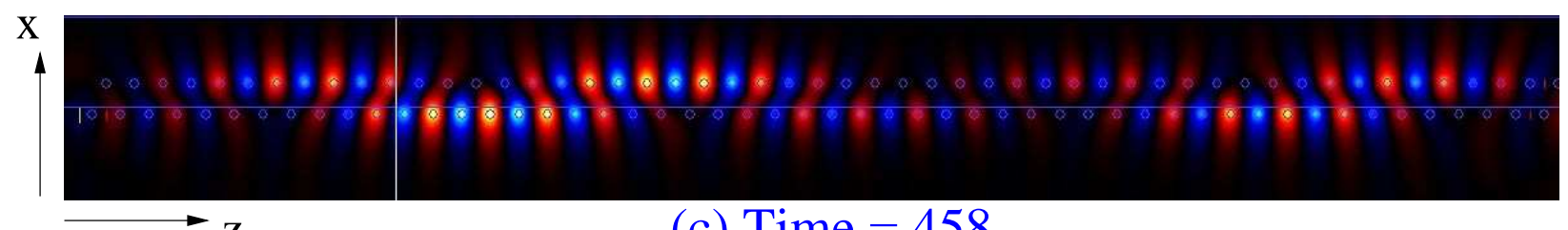

Fig. 6. 
Rods offset 0.35

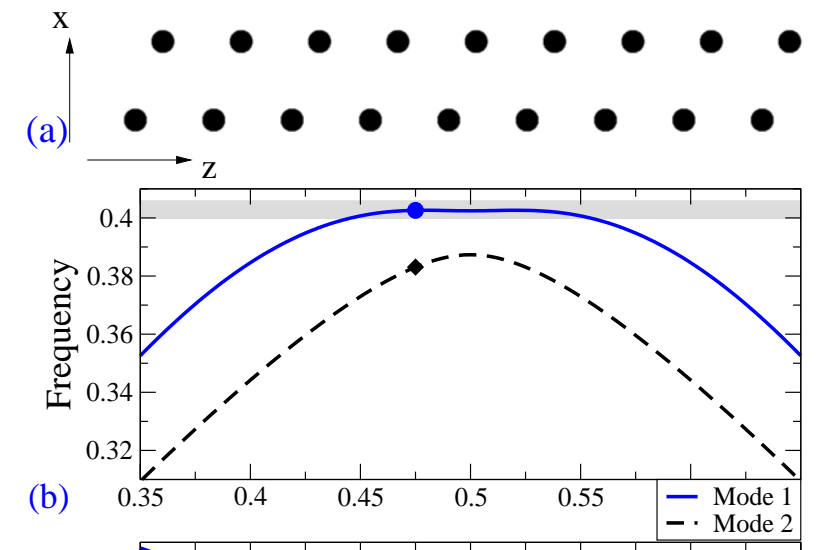

(d)

Mode 1 - Amplitude Phase

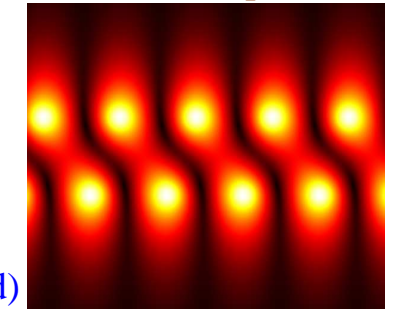

Mode 2 - Amplitude

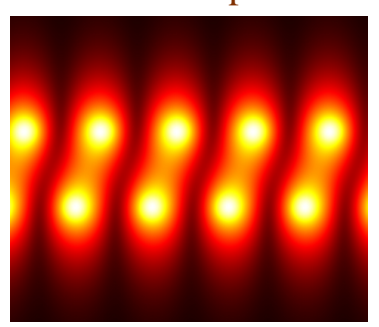

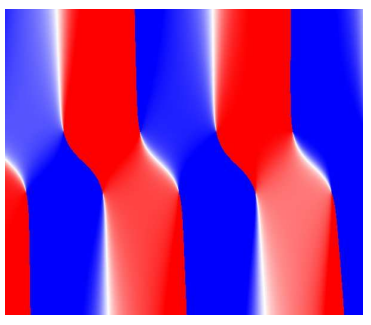

Phase

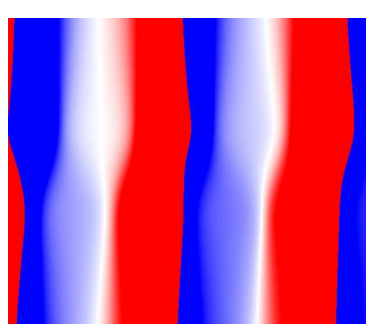

Fig. 7. 
Rods offset 0.35 ; Normalized frequency 0.4029

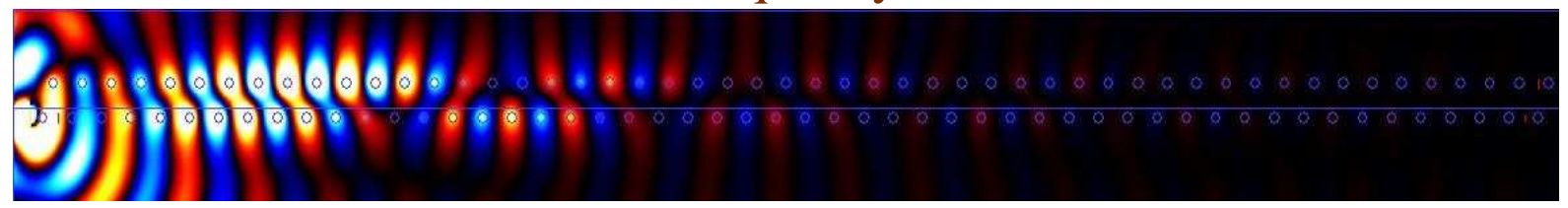

(a) Time $=134$

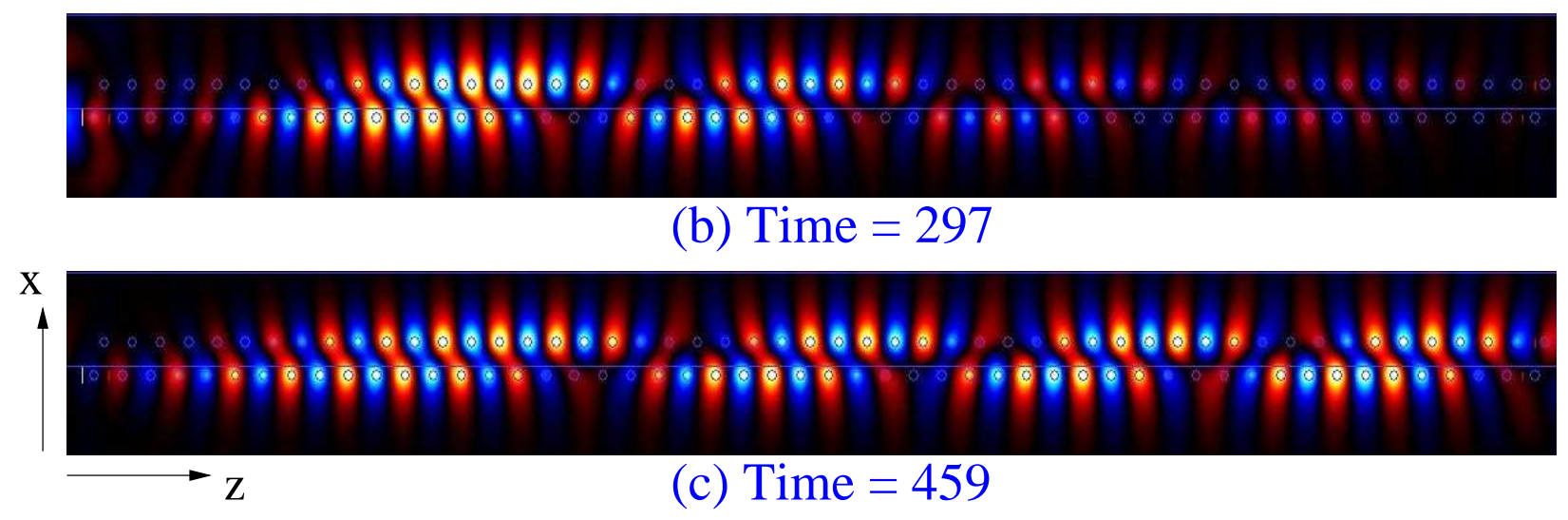

Fig. 8. 
Holes offset 0

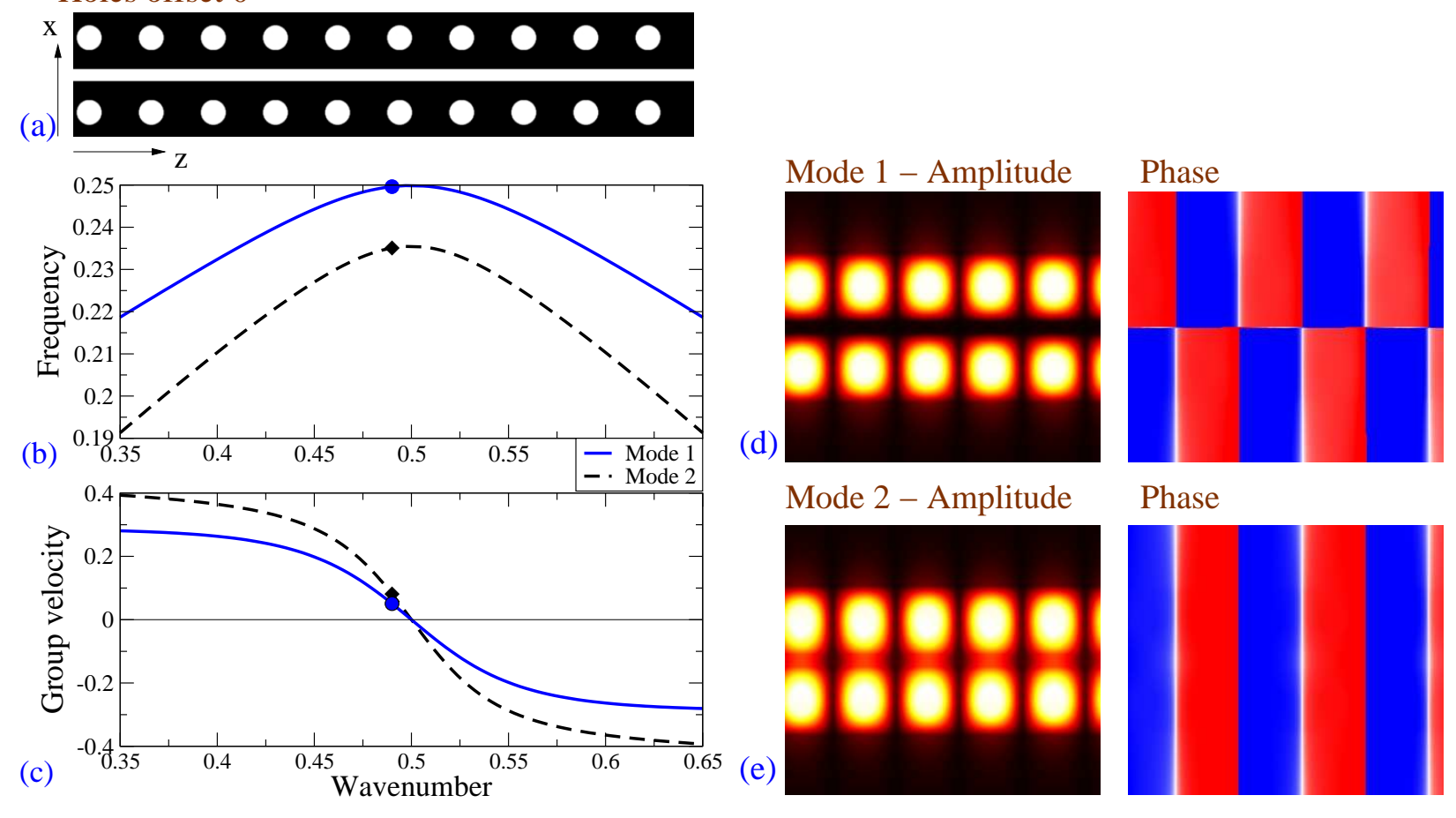

Fig. 9. 
Holes offset 0.28

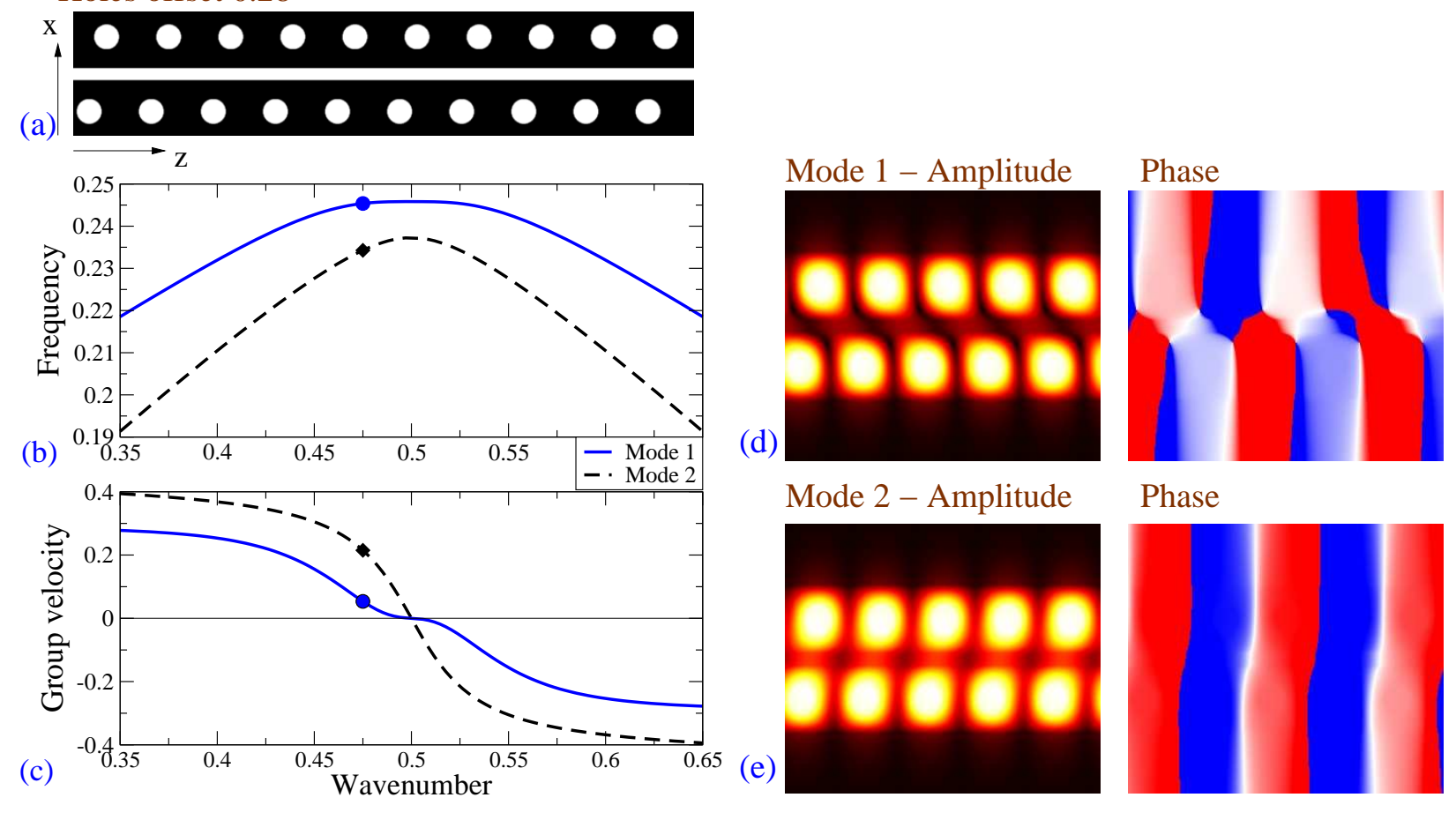

Fig. 10. 
Holes offset 0.5

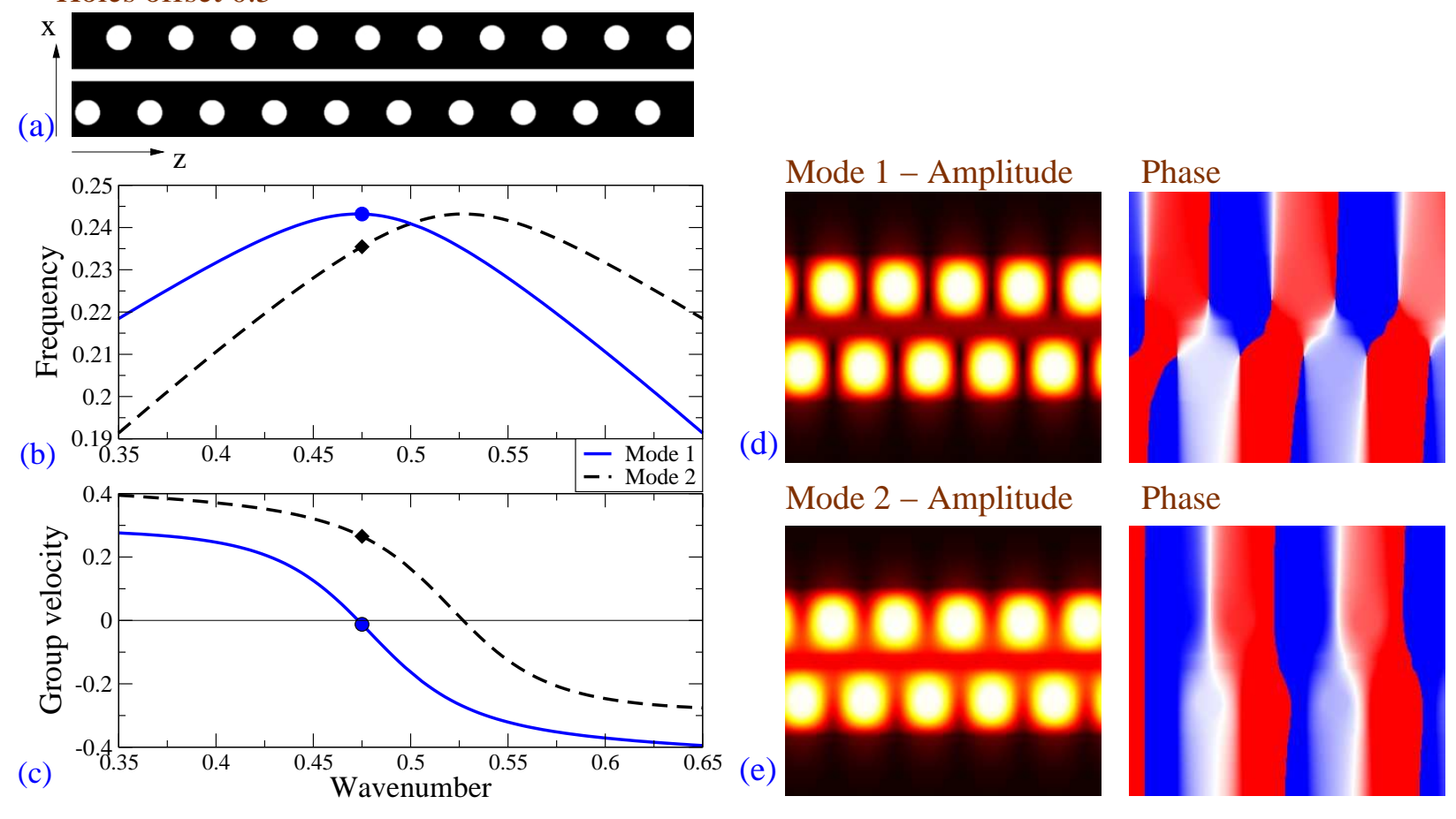

Fig. 11. 